\title{
Evaluation of Procalcitonin Accuracy for the Distinction Between Gram-Negative and Gram-Positive Bacterial Sepsis in Burn Patients
}

\author{
Luís Cabral, $\mathrm{MD},{ }^{*}, \dagger, \odot$ Vera Afreixo, PhD, ${ }^{\ddagger}$ Rita Meireles, $\mathrm{MD},{ }^{*}$ Miguel Vaz, $\mathrm{MD},{ }^{*}$ João-Gonçalo Frade, $\mathrm{PhD}, \|, \$$ \\ Catarina Chaves, MD, "l Marisa Caetano, PharmD, ${ }^{\mathbb{I}}$ Luís Almeida, PhD, ${ }^{* *}$ and José-Artur Paiva, PhD, †t, $\neq \ddagger$
}

\begin{abstract}
Sepsis is the main cause of death in burns. Early institution of antimicrobial therapy is crucial to optimize outcomes but superfluous therapy increases adverse events, microbial resistance, and costs. Blood cultures are the gold standard for diagnosis but can take $\mathbf{4 8}$ to $\mathbf{7 2}$ hours. Biomarkers are used to help sepsis diagnosis and distinction between Gram-negative and Gram-positive bacterial cause. The aim of this work is to evaluate procalcitonin (PCT) accuracy for this distinction in burn patients. Retrospective observational study of adult septic burn patients with $\geq 15 \%$ total burn surface area admitted from January 2011 to December 2014 at a Burn Unit in Portugal. A statistical analysis was done, evaluating the correlation between PCT levels on the day of the first positive blood culture and microbiological data for Gram-negative and Grand-positive bacteria. Patients with mixed bacterial and/or fungal blood cultures were excluded. Data were summarized by quartiles statistics. Blood cultures were positive in 189 patients: 75 (39.7\%) showed growth for Gram-negative and $114(60.3 \%)$ for Gram-positive bacteria. Patients with Gram-negative bacteria have significantly higher PCT levels. Receiver operating characteristic curve analysis showed accuracy for Gram-negative discrimination with area under the curve $=\mathbf{0 . 6 8 7}$. Most elevated levels were related to nonfermentative Gram-negative bacteria and by Klebsiella pneumoniae and other Enterobacteriaceae. PCT levels were significantly higher in burn patients with Gram-negative sepsis comparing to patients with Gram-positive sepsis and controls. The determination of PCT levels may help the choice of empirical antimicrobial therapy while microbiological culture results are not available, despite not fully ensuring the desirable degree of precision.
\end{abstract}

An early and adequate antimicrobial therapy is the main step for the management of septic patients. ${ }^{1}$ Unfortunately, differential diagnosis between sepsis and the systemic inflammatory response triggered by trauma is difficult, particularly in burn patients where the usual clinical signs of sepsis are frequently present even in the absence of microbial infection. ${ }^{2}$ For instance, burn injuries leading to upregulation

\footnotetext{
*Department of Plastic Surgery and Burns Unit, Coimbra University Hospital Centre (CHUC), Portugal;

${ }^{+}$Autonomous Section of Health Sciences (SACS), University of Aveiro, Portugal;

${ }^{\ddagger}$ CIDMA-Center for Research and Development in Mathematics and

Applications, iBiMED-Institute for Biomedicine, Department of Mathematics, University of Aveiro, Portugal;

"Clinical Pathology Department, Coimbra University Hospital Centre (CHUC), Portugal;

${ }^{\$}$ Escola Superior de Saude, Instituto Politécnico de Leiria, Portugal;

"Pharmacy Department, Coimbra University Hospital Centre (CHUC), Portugal;

** Medin UP, Department of Pharmacology and Therapeutics, Faculty of Medicine, University of Porto, Portugal;

${ }^{+}$Department of Emergency and Intensive Care Medicine, Centro Hospitalar São João, Porto, Portugal;

${ }^{\ddagger}$ Grupo de Infecção e Sépsis, Faculty of Medicine, University of Porto, Portugal
}

Funding. V.A. work was supported by Portuguese funds through the CIDMACenter for Research and Development in Mathematics and Applications by the Portuguese Foundation for Science and Technology (FCT-Fundação para a Ciência e a Tecnologia), within project ID/MAT/04106/2013. On behalf of all the other authors, the corresponding author states that none of them has received any funding for this work.

Conflict of interest statement. All authors state that there are no conflicts of interest.

(C) American Burn Association 2018. All rights reserved. For permissions, please e-mail: journals.permissions@oup.com.

doi:10.1093/jbcr/iry058 of the hypothalamic thermal center, physiologic release of catecholamines and cytokines, shift of fluids and the consequent cardiovascular changes, can produce hyperthermia, tachycardia, hypotension, etc., that are transitory and do not reflect any microbial invasion but just a tentative of adjustment of human body systems to the changes in the homeostatic equilibrium.

Authors' Contributions. All authors have read the manuscript and agreed to its content, are accountable for all aspects of the accuracy and integrity of the manuscript in accordance with ICMJE criteria and gave their consent for publication. L.C., V.A., C.C., and M.C. designed the study, interpreted data, and draft the manuscript. V.A. was responsible for most of statistical analysis. R.M., M.V., and J.G.F. were responsible for data acquisition, search of literature, and made suggestions for its integration along the manuscript. C.C. made also substantial intellectual contributions for the Introduction and Discussion sections of the manuscript. L.A. and J.A.P. review the manuscript, and made useful suggestions for Discussion and Conclusions sections.

Ethics Approval and Consent to Participate. Being a retrospective observational study of patients from an anonymized dataset, involving only recording data from the medical record, the Ethics Committee from Coimbra University Hospital Centre (CHUC), waived the need of informed consent according to Declaration of Helsinki and CIOMS International Ethics Guidelines.

Consent for Publication. As all data was anonymized, this study does not contain any individual person's data in any form (including individual details, images, or videos) and accordingly consent for publication was waived.

Availability of Data and Material. The data that support the findings of this study are available from the datasets of the Informatics Department of Coimbra University Hospital Centre but restrictions apply to the availability of these data, which were used under license for the current study, and so are not publicly available. Data are, however, available from the authors, upon reasonable request and after permission of the Ethics Committee from Coimbra University Hospital Centre.

Address correspondence to Luis Cabral, MD, Unidade de Queimados-CHUC Av. Bissaya Barreto s/n, 3000-075 Coimbra Portugal. Email: jlacabral@ gmail.com 
The gold standard for sepsis diagnosis still relies on the microbiological growth in blood cultures, ${ }^{3}$ which can take as long as 48 to 72 hours, according to different facilities, ${ }^{4}$ and the antimicrobial sensibility tests may be available even later. On the other hand, the adequacy of antimicrobial therapy is obviously related with the appropriateness of the chosen drugs, that is, the selection of the most efficacious drug against the causative microorganism. In practical terms, physicians have to assess the presence of sepsis in a complex clinical setting, with great chance of misdiagnosis (false positive or false negative), and in most cases to wait 2 days to confirm their suspicion, having (or not) prescribed an antimicrobial therapy that may be inefficacious against the causative bug, allowing the septic process to progress and increasing the likelihood of a fatal outcome. Moreover, a superfluous or an inappropriate antimicrobial therapy presents risks of adverse events for the patient and stimulates the development of microbial resistance, besides increasing costs. ${ }^{5}$ In conclusion as described in a seminal work by Kumar et al, ${ }^{6}$ it is of outstanding importance the prompt institution of an effective antimicrobial therapy, avoiding the serious risks present when this is not timely done.

In the last decade, biomarkers have been employed to help sepsis diagnosis and antimicrobial prescription and stopping. Together with infection control measures and antimicrobial therapy protocols, the use of biomarkers constitutes the backbone of most antimicrobial stewardship programs. ${ }^{7}$ From a multitude of clinical and biochemical biomarkers described in literature, procalcitonin (PCT) became one of the most employed due to 1) its relatively good accuracy for the diagnosis of septic and nonseptic patients since the first hours of microbial invasion, helping the decision to start or postpone antimicrobial therapy, particularly if used in a dynamic approach; 2) correlation between PCT levels and sepsis severity, and 3 ) its rapid fall when infection is controlled. ${ }^{8}$ Furthermore, significant differences in PCT levels have been found according to the causative pathogens, namely between Gram-negative and Gram-positive bacteria, which facilitates the choice of the drugs to be empirically used meanwhile blood culture results and sensibility tests are not available.

Even being controversial for some authors, determination of PCT serum levels has been consistently advocated for the diagnosis, prognosis, and antimicrobial stewardship in burn patients. Taking into account the different therapeutic approach to different pathogens, it is worthwhile to evaluate the discriminative potential of PCT to set the more appropriate empirical therapy. The aim of this work is to size up PCT performance for the differential diagnosis between sepsis by Gram-negative and Gram-positive bacteria in a large sample of burn patients.

\section{MATERIALS AND METHODS}

\section{Patient Informed Consent}

Considering that this was an observational study using anonymized retrospective data, the Independent Ethics Committee (Comissão de Ética para a Saúde, Coimbra Hospital University Center-CHUC, Coimbra, Portugal) waived the need of informed consent.

\section{Study Plan}

Data for this retrospective observational study was collected from the clinical files and laboratory electronic records of consecutive burn patients with $15 \%$ or more of total burn surface area, admitted from January 2011 to December 2014 at Coimbra Burn Unit (CBU), a department of CHUC. All the patients had positive blood cultures and clinical diagnosis of sepsis, following the American Burn Association criteria9: suspicion of infection coupled with the presence of three or more of the following parameters: temperature $>39^{\circ} \mathrm{C}$ or $<36.5^{\circ} \mathrm{C}$; tachycardia $>110$ beats $/ \mathrm{min}$; tachypnea $>25$ breaths/ min or ventilation $>12 \mathrm{l} / \mathrm{min}$; thrombocytopenia $<100,000 /$ $\mathrm{ml}$; hyperglycemia (untreated plasma glucose $>200 \mathrm{mg} / \mathrm{dl}$ or intravenous glucose requirement $>7 \mathrm{U} / \mathrm{h}$ over 24 hours; enteral feeding intolerance: abdominal distension or gastric residuals more than two times feeding rate or diarrhea $>2500 \mathrm{ml}$.

Blood cultures were obtained in a standardized way. Three samples were collected by sterile venepuncture in septic patients. Except when immediate antimicrobial therapy has to be initiated due to sound clinical or laboratorial sepsis suspicion, the collects were done in the morning (7-8 am). This collect was repeated every 2 days until clinical resolution and PCT normalization.

Using sample patients who never developed sepsis during its stay at $\mathrm{CBU}$ as controls, a statistical analysis was done to evaluate possible correlation of PCT levels on the day of the collection of the first positive blood culture with microbiological data, according to two groups of microorganisms: Gramnegative and Grand-positive bacteria. To avoid potential bias and simplify the analysis, patients with positive mixed bacterial and/or fungal blood cultures were excluded from the study. When a patient had more different microorganisms present in the blood cultures at different timepoints, only PCT levels of the first identification were subjected to analysis. If a patient had more than a PCT measurement on the day of collection, the highest value was used for the analysis. PCT was measured with TRACEC (time-resolved amplified cryptate emission) technology (Kryptor@ PCT; Brahms@ AG; Hennigsdorf, Germany).

\section{Statistical Analysis}

Data were summarized by quartiles statistics. The quantitative variables under study showed a non-Gaussian distribution and thus a nonparametric approach (Kruskall-Wallis and Mann-Whitney tests) was used to compare quantitative variables. Qualitative variables were compared with the Pearson chi-square test. For pairwise comparisons, the Bonferronicorrection was applied.

Receiver operating characteristic (ROC) curves, in particular the area under the curve (AUC), were performed to evaluate PCT ability in Gram-negative and Gram-positive discrimination. Sensitivity, specificity, positive and negative predictive values were calculated for some cutoff values including the best cutoff defined by the maximum value of Youden index $(J=$ sensitivity + specificity -1$)$.

Statistical analysis was performed with SPSS 25.0 IBM( for Windows $($ ). A $p$ value of less than .05 was set as the level of significance and the confidence intervals are reported with $95 \%$ confidence level. 


\section{RESULTS}

The sample under analysis was composed 438 burn patients. Among these patients, 249 (56.8\%) did not fulfill American Burn Association sepsis criteria neither had any growth in their blood cultures during their stay at CBU, being deemed to serve as controls. Blood cultures were positive in 189 (43.2\%) patients; among from these, 75 patients (39.7\%) showed growth for Gram-negative bacteria and 114 (60.3\%) showed growth for Gram-positive bacteria (Table 1). The median age was 62 years for controls, 66 years for patients with sepsis by Gram-negative bacteria and 69 years for patients with sepsis by Gram-positive bacteria; the difference among groups did not reach statistical significance. The same was true for gender distribution, which showed a preponderance of the masculine sex: control patients included 152 males $(61 \%)$ and 97 females (49\%); the Gram-negative group was composed by 41 males $(55 \%)$ and 34 females (45\%) meanwhile the Gram-positive group gathered 70 males (61\%) and 44 females (39\%; Table 1).

On the day of the first identification of microbiological growth in blood cultures, PCT levels were significantly higher in patients with Gram-negative bacteria comparing to controls and patients with Gram-positive bacteria; the differences between controls and Gram-positive infected patients did not reach statistical significance (Table 2).

Figure 1 depicts box-plots for PCT levels in the first day of microbiological identification, clearly showing higher values for patients in the Gram-negative group in relation to control group and to Gram-positive group while the difference between these later groups is not evident.

The maximum value of the Youden index was 0.31 , for a cutoff $=0.57 \mathrm{ng} / \mathrm{ml}$. This cutoff reached a sensibility of $63 \%$ and a specificity of $68 \%$; the corresponding positive predictive value was set in $57 \%$ and the corresponding negative predictive value achieved $74 \%$ (Table 3 ). This was the optimum PCT cutoff, corresponding to the maximum point of the ROC curve: higher ones were associated with lesser sensitivity and lower ones led to loss of specificity.

ROC curve is presented in Figure 2. The AUC showed a significant accuracy for Gram-negative discrimination from Gram-positive: AUC $=0.687$, with $95 \%$ confidence interval $=0.609-0.765$.

Subgroup analysis was performed including the most frequent Gram-negative and Gram-positive microorganisms responsible for sepsis in this sample of patients. In the Gram-negative group, the mostly frequently isolated agent was Pseudomonas aeruginosa, as it would be expected according to its great prevalence in many burn units, followed by Acinetobacter spp. and other nonfermentative bacteria, including Burkholderia cepacia and Stenotrophomonas maltophilia. The Enterobacteriaceae were also very common, namely Escherichia coli, Enterobacter spp., Klebsiella pneumonia, Serratia marcescens, Proteus mirabilis, etc. From the Gram-positive group, Staphylococcus epidermidis, Staphylococcus hominis and other coagulase-negative species of Staphylococci, some of them without more specific identification furnished by the laboratory, were the most isolated from the blood samples. As it happened with Bacillus spp. and Corynebacterium spp., most of times the coagulase-negative species of Staphylococci species were suggested to be probable contaminants in the microbiological results and sensibility tests. Group D Enterococci (namely Enterococcus faecalis and Enterococcus faecium) and Staphylococcus aureus were also very frequently isolated and there were also isolations of Streptococcus spp. Table 4 displays the list of the most common microorganisms and the corresponding values of PCT levels on the first day of microbiological identification. The full list can be found in Supplementary Annex I.

Despite the presence of several outliers, it was found that PCT levels in the Gram-negative group were in general significantly higher comparing to controls, what did not happen in the Gram-positive group, with the exception of patients with sepsis due to Streptococcus spp. (Figure 3). With the exception of those with sepsis due to this Gram-positive species, which isolation is rare ate CBU, in almost all patients with PCT concentrations above $3.00 \mathrm{ng} / \mathrm{ml}$ on the day of collection of the first positive blood culture, the causative microorganism was a Gram-negative agent.

In the first case, the statistical difference was more pronounced for glucose nonfermenting bacilli (particularly Acinetobacter, Pseudomonas, and Burkholderia spp.) and for E. coli and K. pneumoniae, glucose fermenting rods from the Enterobacteriaceae family. Among patients with sepsis due to Gram-positive cocci, PCT levels only reach statistically significant difference for Streptococcus spp., as referred, but there was a trend for significance for Enterococcus spp. and for $S$. aureus (not visible for nonaureus species).

\section{DISCUSSION}

The statistical analysis of PCT levels on the first day of microbiological identification in blood samples in this sample

Table 1. Population characteristics

\begin{tabular}{lccccc}
\hline & & Controls & Gram-Negative Sepsis & Gram-Positive Sepsis \\
\hline Number of Patients & & 249 & 75 & 114 \\
& Median & 62.0 & 66.0 & 69.0 \\
Age (years) & Q1-Q3 & $45.5-78.0$ & $44.5-79.5$ & $47-80.0$ & $70(61 \%)$ \\
Sex & Males & $152(61 \%)$ & $41(55 \%)$ & $44(39 \%)$ & $.392^{*}$ \\
Procalcitonin & Females & $97(49 \%)$ & $34(45 \%)$ & 0.32 & $.578^{\dagger}$ \\
\multicolumn{1}{c}{$(\mathrm{ng} / \mathrm{ml})$} & Median & 0.20 & $0.35-4.15$ & $0.16-0.87$ \\
\hline
\end{tabular}

* Kruskall-Wallis test.

†Chi-square test. 
Table 2. Pairwise comparisons for procalcitonin levels between sepsis groups

\begin{tabular}{lc}
\hline Comparison & $P$ \\
\hline Gram-negative septic patients vs controls & .000 \\
Gram-negative septic patients vs Gram-positive septic patients & .000 \\
Gram-positive septic patients vs controls & .153
\end{tabular}

Mann-Whitney test with Bonferroni-corrected $P$-values.

of extensively burned patients confirmed previous reports demonstrating significantly higher values in the presence of Gram-negative bacteria comparing with controls or patients with Gram-positive sepsis. ${ }^{10-13}$ The difference was most pronounced when causative agents were glucose nonfermenting bacilli, particularly Acinetobacter and Pseudomonas spp., or Enterobacteriaceae rods, like E. coli or K. pneumoniae. On the other hand, a statistical difference in PCT levels was not found between in PCT levels of patients with sepsis caused by Gram-positive bacteria and control patients, with the exception of patients with sepsis caused by Streptococcus spp.

The results of this work are consistent with medical literature. Opal and Cohen ${ }^{14}$ attributed the different characteristics of sepsis caused by Gram-negative and Gram-positive to the different constitution of their respective cell membranes, which will trigger different immunological responses and are, in most part, correlated with diverse clinical presentations and outcomes. ${ }^{15}$ Briefly explaining, despite there is not yet a full understanding of the mechanisms involved in cytokines activation following microbial insult, it is consensual that human innate immune cells (macrophages, neutrophils, dendritic cells) have receptors, present either on the external cell membrane or inside the cytoplasm (endosomes) which are apt to recognize specific circulating molecular patterns. These pattern recognition receptors (PRRs) can be activated by molecular patterns resulting from nonmicrobial tissue damage (damage-associated molecular patterns, DAMPs) or by those exclusively corresponding to microbial pathogenic components (pathogen-associated molecular patterns, PAMPs). ${ }^{16}$ The interaction between PRRs and PAMPs induces the release of cytokines by immune cells, initiating the septic process.

There are several types of PRRs, including Toll-like receptors (TLRs) and NOD-like receptors-mainly activated by bacteria; RIG-I-like receptors and DNA-sensing molecules-crucial for sensing of viruses; C-type lectin receptors responding to fungi and mycobacteria PAMPs; etc. The outer membrane of Gram-negative bacteria cell wall is composed mostly by lipopolysaccharide, frequently referred as endotoxine, which is its principal PAMP, being recognized by TLR $4 .{ }^{17}$ Instead of lipopolysaccharide, PAMPs of Gram-positive bacteria cell wall are basically lipoteichoic acid, ${ }^{18}$ lipoproteins and proteoglicans, mostly sensed by TLR 2 .

TLR4 activation triggers a strong release of inflammatory cytokines, namely tumor-necrosis factor $\alpha$, interleukin-1, and interleukin-6. ${ }^{19}$ These cytokines will promote gene transcription leading to PCT secretion from extrathyroidal tissues, with abrupt rise of its blood levels. It was also described a direct stimulation of PCT secretion by circulating endotoxins. ${ }^{20} \mathrm{On}$ the other hand, TLR2 activation usually induces a relatively weaker and not always straightforward production of those cytokines, varying according to different pathogens by not well known reasons.

In 2008, Charles and colleagues analyzed the accuracy of PCT measurements to discriminate between Gram-negative and Gram-positive bacteremia at the onset of bloodstream infection, concluding that serum levels were greater in the

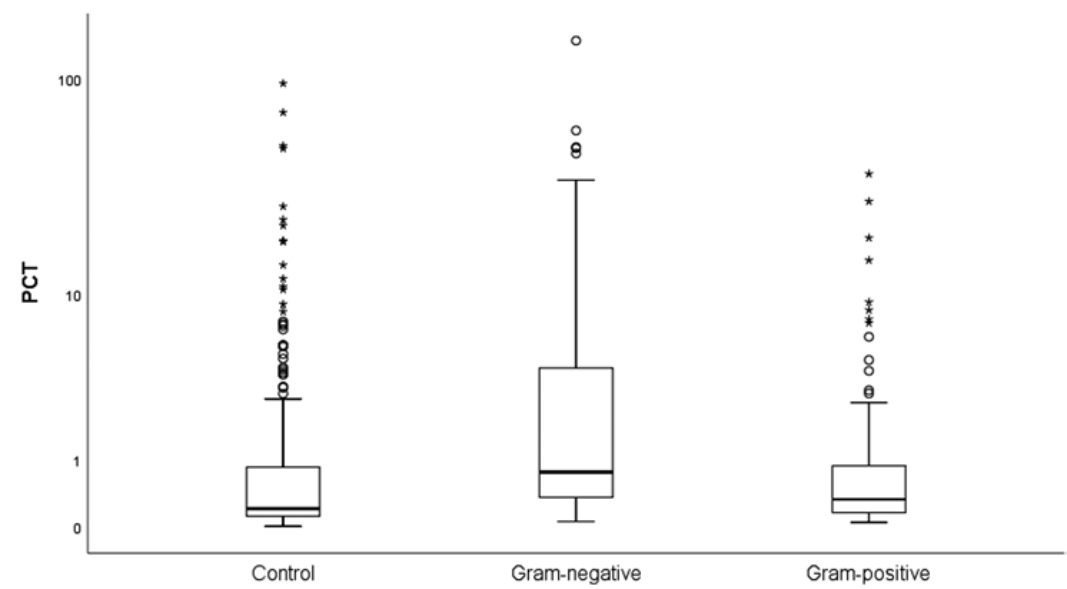

Figure 1. Box-plots for procalcitonin levels in controls $(n=249)$, Gram-negative $(n=75)$, and Gram-positive $(n=114)$ sepsis patients groups.

Table 3. Sensitivity, specificity, positive predictive value (PPV), and negative predictive value (NPV) of procalcitonin cutoffs for the distinction between Gram-negative and Gram-positive sepsis in burn patients

\begin{tabular}{lcccccr}
\hline Cutoff & Sensitivity (\%) & Specificity (\%) & PPV (\%) & NPV (\%) & Youden & Obs. \\
\hline 0.50 & 64 & 62 & 53 & 72 & 0.26 & Max. Youden \\
0.57 & 63 & 68 & 57 & 74 & 0.31 & 0.22 \\
1.00 & 46 & 52 & 62 & 68 & 0.15 \\
5.00 & 23 & 92 & & & \\
\hline
\end{tabular}




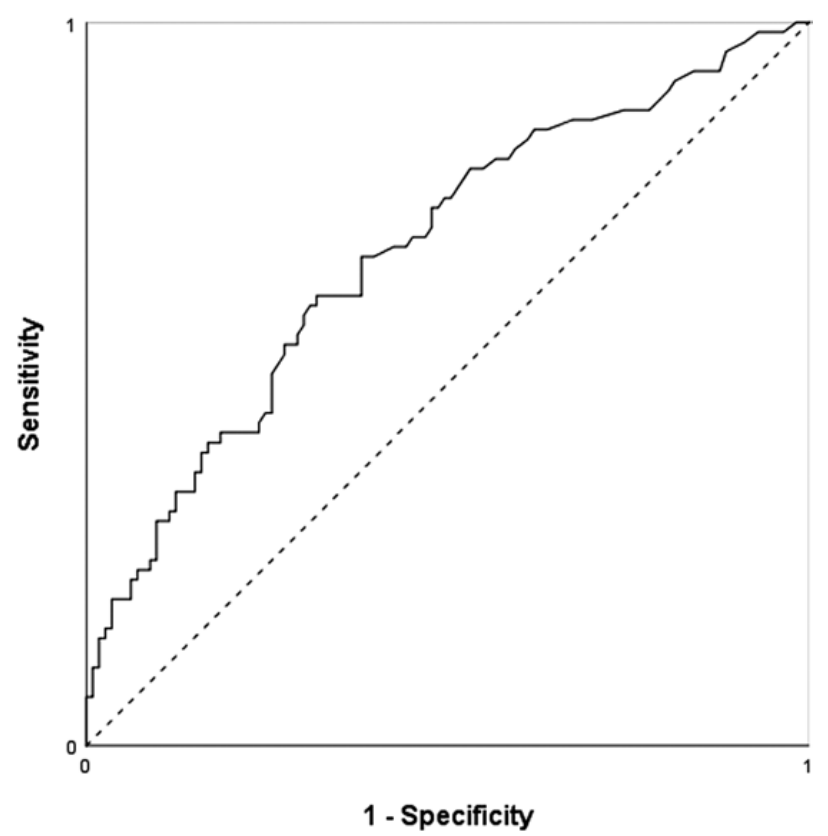

Figure 2. Receiver operating characteristic curve for procalcitonin accuracy in the distinction between Gram-negative and Gram-positive bacterial sepsis in burn patients (cutoff $=0.57 \mathrm{ng} / \mathrm{ml}$ ).

first group, with an AUC of 0.79 , opposing to what happened with the measurements of C-reactive protein and leucocyte counting. ${ }^{21}$ As PCT levels determination is available sooner than Gram stain results and microbiological identification, these authors suggest this information should be taking in account when choosing empirical antibiotherapy for sepsis.

In 2012, Jeong et al, ${ }^{22}$ showed a good performance of PCT in the distinction between patients with negative and positive blood cultures, facilitating the differentiation of true bloodstream infections from contamination. They also reported that significantly higher values were found for Gram-negative bacteremia comparing to Gram-positive or fungal ones, what did not happen with C-reactive protein values. With most patients coming from hematological ICUs, an article by Brodská et $\mathrm{al}^{23}$ described significantly higher PCT values for patients with Gram-negative bacterial infections, comparing to Gram-positive or fungal infections, meanwhile no statistical difference was found between these latter two groups. They concluded that PCT levels could be used to help the confirmation or exclusion of Gram-negative sepsis. Nakajima et al, ${ }^{24}$ presented similar results in 2014, speculating the possibility of using PCT levels to help antimicrobial empiric antibiotherapy.

In 2015, Oussalah et $\mathrm{al}^{25}$ using a comprehensive electronic database, performed an observational cross-sectional study and analyzing 2699 patients with positive blood cultures, found statistically higher PCT levels in patients with Gramnegative sepsis comparing patients with Gram-positive sepsis, with most elevate values for Escherichia spp., Bacteroides spp., Klebsiella spp. and Enterobacter spp. They also pointed values under $0.75 \mathrm{ng} / \mathrm{ml}$ as very effective for exclusion of most clinical relevant pathogens, meanwhile a cutoff above $10 \mathrm{ng} / \mathrm{ml}$ practically excluded the hypothesis of sample contamination or fungal infection. In a prospective study, including 1949 adult patients with positive blood cultures, Leli et $\mathrm{al}^{26}$ also reported significantly higher PCT levels for Gram-negative infections, more pronounced for Enterobacteriaceae bacteria, suggesting a cutoff of $3.1 \mathrm{ng} / \mathrm{ml}$ for the exclusion of these microorganisms. Guo et $\mathrm{al}^{27}$ reached the same results in a sample of 280 septic patients and listed Klebsiella, Escherichia, Acinetobacter, Enterobacter, and Pseudomonas as the pathogenic species responsible for higher PCT levels. In 2016, Li et al ${ }^{28}$ analyzing 328 septic episodes, suggested that PCT levels might be used as a surrogate marker to distinguish sepsis cases originated by Gram-negative bacteria from the ones deriving from Gram-positive bacterial or fungal invasion of bloodstream, proposing a cutoff of $2.44 \mathrm{ng} / \mathrm{ml}$. Yan et $\mathrm{al}^{29}$ reviewed data from 484 monomicrobial positive blood cultures of septic patients $(75 \%$ collected at the ICU and $25 \%$ at the Emergency Department), reporting statistically significant differences in PCT levels, with higher values corresponding to patients

Table 4. Procalcitonin values for the most frequently isolated groups of microorganisms in blood samples of septic burn patients

\begin{tabular}{|c|c|c|c|c|c|}
\hline Microorganism & Number & Median & Q1 & Q3 & $P$ \\
\hline Controls & 249 & 0.20 & 0.11 & 0.84 & \\
\hline \multicolumn{6}{|c|}{ Glucose nonfermenting Gram-negative Bacilli } \\
\hline Acinetobacter spp. & 13 & 1.17 & 0.49 & 7.30 & .002 \\
\hline Pseudomonas spp. & 13 & 0.67 & 0.39 & 1.68 & .005 \\
\hline Burkbolderia cepacia & 4 & 1.82 & 0.89 & 3.05 & .045 \\
\hline Xanthomonas maltophilia & 4 & 0.63 & 0.29 & 8.89 & .241 \\
\hline \multicolumn{6}{|l|}{ Enterobacteriaceae } \\
\hline Enterobacter & 9 & 0.55 & 0.22 & 0.62 & .087 \\
\hline Escherichia coli & 5 & 2.96 & 0.75 & 6.90 & .020 \\
\hline Klebsiella pneumoniae & 5 & 1.77 & 0.58 & 22.18 & .043 \\
\hline Serratia marscecens & 5 & 0.75 & 0.48 & 0.89 & .255 \\
\hline \multicolumn{6}{|l|}{ Gram-positive Cocci } \\
\hline Enterococcus spp. & 12 & 0.38 & 0.18 & 0.73 & .177 \\
\hline Staphylococcus aureus & 11 & 0.28 & 0.21 & 0.97 & .185 \\
\hline Staphylococcus (except aureus) & 54 & 0.29 & 0.11 & 0.88 & .668 \\
\hline Streptococcus spp. & 8 & 2.18 & 1.27 & 4.91 & .003 \\
\hline
\end{tabular}




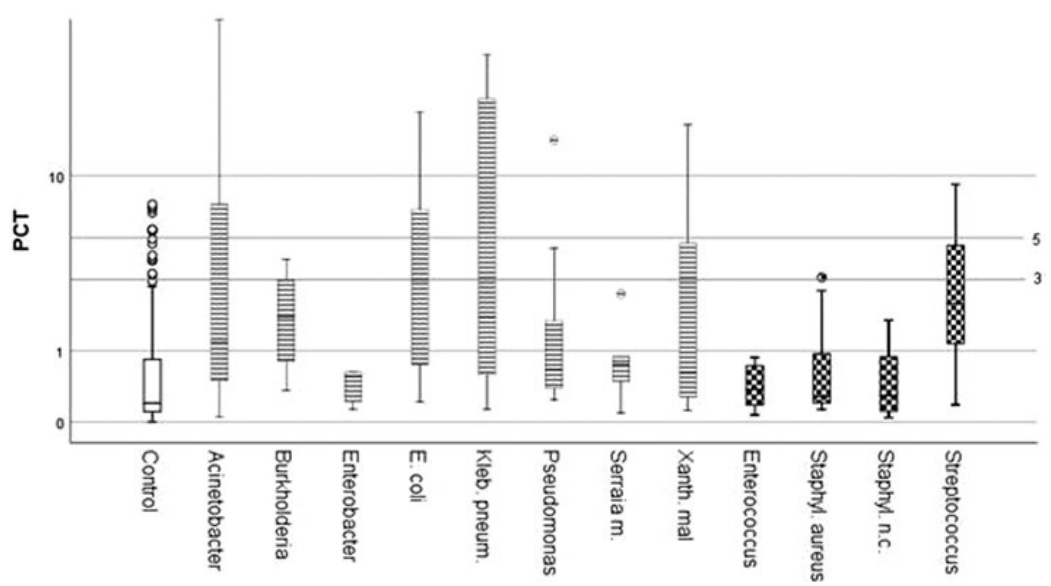

Figure 3. Box-plots for procalcitonin levels in Gram-negative and Gram-positive bacterial sepsis subgroups.

with Gram-negative infection. From the Gram-negative bacterial sepsis group, PCT levels were more pronounced for Enterobacteriaceae microorganisms (E. coli, Kebsiella spp., Enterobacter spp., and Serratia spp., by this order), which presented relatively higher values than glucose nonfermenting Gram-negative bacilli ( $P$. aeruginosa, Acinetobacter baumanii, S. maltophilia, B. cepacia, etc.). From the Gram-positive bacterial sepsis group, patients infected by Streptococcus spp., Enteroccoccus spp., and $S$. aureus had the most elevated PCT concentrations. The authors defended that PCT could be useful not only to distinguish between Gram-negative and Grampositive sepsis, but might even be employed to identify diverse species inside each of these groups of microorganisms.

In a work from 2018, Thomas-Rüddel et al performed a secondary analysis of a prospectively collected dataset, including a very large sample with 4858 septic patients from 40 hospitals. Their results were very similar to the present study, showing distinctly higher values for PCT concentrations in patients with Gram-negative bacteremia than in patients with sepsis resulting from Gram-positive or fungal systemic invasion. ${ }^{30}$ Indeed, the AUC for the discrimination of Gram-negative sepsis from Gram-positive was identical, is spite substantially diverse cutoffs. Subgroups of pathogens with the most elevated values were also very close, with Streptococcus spp.; E. coli, Proteus spp., K. pneumoniae and other Enterobacteriaceae on the top. The authors referred, however, a large overlap of PCT levels and speculate that higher values may be more related with higher bacterial load and potentially with intrinsic characteristics of pathogens groups, considering the discriminatory power too low to guide therapeutic decisions.

Burn patients have a risk of infection superior to the average critical care patient and sepsis diagnosis is more difficult ${ }^{31}$ due to the intense inflammatory systemic response unleashed by the burn insult per se. In these patients, PCT measurements, particularly using a kinetic approach, have being increasingly advocated by many authors to help the differentiation between pure inflammatory reaction and microbial infection $^{32-36}$ and for antimicrobial stewardship. ${ }^{37}$ However, this strategy is still not fully accepted ${ }^{38-42}$ in spite of systematic reviews and meta-analysis suggesting its validity. ${ }^{43-45}$ In 2012, a study of Lavrentieva et al, ${ }^{46}$ including 86 burn patients, was presumably the first work reporting statistically significant differences of PCT levels between burn patients with Gramnegative sepsis and those with Gram-positive sepsis, with the most elevated values in the former group. Mokline et al in a paper of $2015,{ }^{47}$ including 44 patients, confirmed these results.

To the authors' knowledge, the present work, with 189 septic burn patients, from a homogenous population, corresponds to the largest sample already analyzed in medical literature regarding this subject. It confirms previous reports and, moreover, it further details subgroups differences. On its strengths one can also count the use of strict and internationally validated criteria for definition of burn sepsis, as well as the exclusive utilization of microbiological positive bloodstream cultures, collected in a standardized way, avoiding potential bias due to the use of other types of biological samples. The results of this study, with PCT showing a fair capacity for the distinction between Gram-negative and Gram-positive sepsis insinuate the possibility of using its values in face of sound suspicion of sepsis in burn patients to help the choice of empirical therapy until definitive microbiological identification is available. Cutoffs will be clearly dependent on the idiosyncratic characteristics from each facility, depending on its nosocomial flora and its patients and cannot be generalizable. However very high PCT levels (for instance, above 3.00 or $5.00 \mathrm{ng} / \mathrm{ml}$ ) would usually be more associated with Gram-negative sepsis, with fair positive predictive value and negative predictive value, and good specificity in spite outliers may be present. Also, in the great majority of the cases, PCT values under $0.5 \mathrm{ng} / \mathrm{ml}$ will not correspond to Gram-negative infections but to Gram-positive or fungal ones.

Paying attention that the majority of deaths in burn patients result from infectious episodes and sepsis ${ }^{48}$ is clear that prompt, adequate and appropriate selection of antimicrobial therapy is crucial for the outcome of these high risk patients. On the other hand, potential damages from adverse events and the contribution to the development of microbial resistance due to superfluous antimicrobial therapy must be duly considered. Meanwhile faster methods of microbiological identification, such as polymerase chain reaction, ${ }^{49}$ mass spectrometry ionization (Matrix-Assisted Laser Desorption Ionization-Time of Flight), gene expression profiling, aptamers panels, etc. ${ }^{50}$ or even more sophisticated and individualized system-based ones (integrating genomics, metabolomics, and proteomics data), are not either widely available or fully developed, ${ }^{51-53}$ PCT dosing will remain one of the more useful tools to help clinicians decisions. . $^{5,54,55}$ 
For these reasons, in the authors' opinion, it is worthwhile to use PCT measurements to have a more empowered prescription decision, even bearing in mind that the analysis of its levels does not fully ensure the desirable degree of precision.

The present work has manifestly some limitations that should be noticed. First, being a retrospective study it is more prone to selection bias than a prospective one. On the other hand, all patients enrolled came from the same center, so the results obtained may not be exactly reproduced in other Burn Units. Subgroup analysis according to associated pathologies was not done, neither the results from other current biomarkers like CRP or leucocyte counting were noted. However, according to the available literature, the relevance of these biomarkers is at least very questionable for the purposes of this study. Due to the small number of positive blood samples with fungi found during the study period, comparison with PCT levels in Gram-negative and Gram-positive sepsis was not done. To avoid confusion, mixed infections were purposely not included. It would also had been very interesting to further extend the analysis of PCT levels to the subsequent days after the positivation of blood samples, assessing the potential added value of PCT kinetics regarding distinction of different types of bacterial infection.

\section{CONCLUSIONS}

This retrospective study consistently showed the presence higher PCT levels in burn patients with Gram-negative sepsis, suggesting that PCT may help clinicians in the choice of the empirical antimicrobial therapy, while the definitive, gold standard, microbiological culture results and sensibility tests are not yet available. However, it should be emphasized that PCT must be integrated within the clinical context and the facility prevalent flora, and it can never substitute clinicians' evaluation and judgment. Prospective multicentric studies are needed to get a stronger validation of the use of PCT values for the distinction between Gram-negative and Gram-positive bacterial sepsis and it would be also desirable to include fungal and mixed infections. Evaluation of PCT kinetics potential for differential diagnosis between microbial sepsis due to diverse types of pathogens would also be very interesting and potentially useful for clinical practice.

\section{SUPPLEMENTARY DATA}

Supplementary data is available at Journal of Burn Care \& Research online.

\section{ACKNOWLEDGEMENTS}

The authors are grateful for the support received from Dr Fernando Rodrigues, Director of the Department of Clinical Pathology of Coimbra University Hospital Centre, specially by the prompt availability of the laboratory data used in the study, and also thank the generous help provided by Dr Helena Donato, Director of the Documentation Service of the same hospital, regarding bibliographic research.

\section{REFERENCES}

1. Ibrahim EH, Sherman G, Ward S, Fraser VJ, Kollef MH. The influence of inadequate antimicrobial treatment of bloodstream infections on patient outcomes in the ICU setting. Chest 2000;118:146-55.
2. Stanojcic M, Vinaik R, Jeschke MG. Status and challenges of predicting and diagnosing sepsis in burn patients. Surg Infect (Larchmt) 2018;19:168-75.

3. Mancini N, Carletti S, Ghidoli N, Cichero P, Burioni R, Clementi M. The era of molecular and other non-culture-based methods in diagnosis of sepsis. Clin Microbiol Rev 2010;23:235-51.

4. Nellis ME, Pon S, Giambrone AE, et al. The diagnostic accuracy of serum procalcitonin for bacteremia in critically ill children. Infect Dis Clin Pract (Baltim Md) 2016;24:343-7.

5. Schuetz P, Bretscher C, Bernasconi L, Mueller B. Overview of procalcitonin assays and procalcitonin-guided protocols for the management of patients with infections and sepsis. Expert Rev Mol Diagn 2017;17:593-601.

6. Kumar A, Roberts D, Wood KE, et al. Duration of hypotension before initiation of effective antimicrobial therapy is the critical determinant of survival in human septic shock. Crit Care Med 2006;34:1589-96.

7. Broyles MR. Impact of procalcitonin-guided antibiotic management on antibiotic exposure and outcomes: real-world evidence. Open Forum Infect Dis 2017;4:ofx213.

8. Riedel S. Procalcitonin and the role of biomarkers in the diagnosis and management of sepsis. Diagn Microbiol Infect Dis 2012;73:221-7.

9. Greenhalgh DG, Saffle JR, Holmes JH, et al. American Burn Association consensus conference to define sepsis and infection in burns. J Burn Care Res 2007;28:776-90.

10. Inci A. Investigation of differences in CRP, PCT, WBC and MPV in Gram-negative, Gram-positive and fungal bloodstream infections. Dis Mol Med 2016;4:81-4.

11. Watanabe Y, Oikawa N, Hariu M, Fuke R, Seki M. Ability of procalcitonin to diagnose bacterial infection and bacteria types compared with blood culture findings. Int J Gen Med 2016;9:325-31

12. Irvem A, Aksaray S. Procalcitonin, C-reactive protein, leukocyte, mean platelet volume levels in bloodstream infections. J Clin Anal Med 2018:9:391-5.

13. Bilgili B, Haliloğlu M, Aslan MS, Sayan İ, Kasapoğlu US, Cinel İ Diagnostic accuracy of procalcitonin for differentiating bacteraemic gram-negative sepsis from gram-positive sepsis. Turk J Anaesthesiol Reanim 2018;46:38-43

14. Opal SM, Cohen J. Clinical Gram-positive sepsis: does it fundamentally differ from Gram-negative sepsis? Crit Care Med 1999;27:1608-16.

15. Feezor RJ, Oberholzer C, Baker HV, et al. Molecular characterization of the acute inflammatory response to infections with gram-negative versus gram-positive bacteria. Infect Immun 2003;71:5803-13

16. Chandler CE, Ernst RK. Bacterial lipids: powerful modifiers of the innate immune response. F1000 Faculty Rev 2017;1334.

17. Kumar S, Ingle H, Prasad DV, Kumar H. Recognition of bacterial infec tion by innate immune sensors. Crit Rev Microbiol 2013;39:229-46.

18. Ryu YH, Baik JE, Yang JS, et al. Differential immunostimulatory effects of Gram-positive bacteria due to their lipoteichoic acids. Int Immunopharmacol 2009;9:127-33.

19. Gao H, Evans TW, Finney SJ. Bench-to-bedside review: sepsis, severe sepsis and septic shock - does the nature of the infecting organism matter? Crit Care 2008;12:213

20. Matwiyoff GN, Prahl JD, Miller RJ, Carmichael JJ, et al. Immune regula tion of procalcitonin: a biomarker and mediator of infection with gramnegative versus gram-positive bacteria. Inflamm Res 2012;61:401-9.

21. Charles PE, Ladoire S, Aho S, Quenot JP, et al. Serum procalcitonin elevation in critically ill patients at the onset of bacteremia caused by either gram negative or gram positive bacteria. BMC Infect Dis $2008 ; 8: 38$.

22. Jeong S, Park Y, Cho Y, Kim HS. Diagnostic utilities of procalcitonin and C-reactive protein for the prediction of bacteremia determined by blood culture. Clin Chim Acta 2012;413:1731-6.

23. Brodská $\mathrm{H}$, Malícková $\mathrm{K}$, Adámková $\mathrm{V}$, Benáková $\mathrm{H}$, et al. Significantly higher procalcitonina levels could differentiate Gram-negative sepsis from Gram-positive sepsis and fungal sepsis. Clin Exp Med 2013;13:165-70.

24. Nakajima A, Yazawa J, Sugiki D, et al. Clinical utility of procalcitonin as a marker of sepsis: a potential predictor of causative pathogens. Intern Med 2014:53:1497-503.

25. Oussalah A, Ferrand J, Filhine-Tresarrieu P, et al. Diagnostic accuracy of procalcitonin for predicting blood culture results in patients with suspected bloodstream infection: an observational study of $35,343 \mathrm{con}-$ secutive patients (A STROBE-compliant article). Medicine (Baltimore) 2015;94:e1774

26. Leli C, Ferranti M, Moretti A, Al Dhahab ZS, Cenci E, Mencacci A. Procalcitonin levels in gram-positive, gram-negative, and fungal bloodstream infections. Dis Markers 2015;2015:701480.

27. Guo SY, Zhou Y, Hu QF, Yao J, Wang H. Procalcitonin is a marker of gram-negative bacteremia in patients with sepsis. Am J Med Sci 2015;349:499-504

28. Li S, Rong H, Guo Q, Chen Y, Zhang G, Yang J. Serum procalcitonin levels distinguish Gram-negative bacterial sepsis from Gram-positive bacterial and fungal sepsis. J Res Med Sci 2016;21:39. 
29. Yan ST, Sun LC, Jia HB, Gao W, Yang JP, Zhang GQ. Procalcitonin levels in bloodstream infections caused by different sources and species of bacteria. Am J Emerg Med 2017;35:579-83

30. Thomas-Rüddel DO, Poidinger B, Kott M, Weiss M, Reinhart K, Bloos F; MEDUSA study group. Influence of pathogen and focus of infection on procalcitonin values in sepsis patients with bacteremia or candidemia. Crit Care 2018;22:128.

31. Muñoz B, Súarez-Sánches R, Hernández-Hernández $\mathrm{O}$, Franco Cendejas R et al. From traditional biochemical signals to molecular markers for detection of sepsis after burn injuries. Burns 2018; pii:S03054179(18)30241-9. doi: 10.1016/j.burns.2018.04.016

32. von Heimburg D, Stieghorst W, Khorram-Sefat R, Pallua N. Procalcitonin-a sepsis parameter in severe burn injuries. Burns $1998 ; 24: 745-50$

33. Sachse C, Machens HG, Felmerer G, Berger A, Henkel E. Procalcitonin as a marker for the early diagnosis of severe infection after thermal injury. J Burn Care Rehabil 1999;20:354-60.

34. Lavrentieva A, Kontakiotis T, Lazaridis L, et al. Inflammatory markers in patients with severe burn injury. What is the best indicator of sepsis? Burns 2007;33:189-94

35. Abdel-Hafez NM, Saleh Hassan Y, El-Metwally TH. A study on biomarkers, cytokines, and growth factors in children with burn injuries. Ann Burns Fire Disasters 2007;20:89-100.

36. Barati M, Alinejad F, Bahar MA, et al. Comparison of WBC, ESR, CRP and PCT serum levels in septic and non-septic burn cases. Burns 2008;34:770-4.

37. Cabral L, Afreixo V, Santos F, Almeida L, Paiva JA. Procalcitonin for the early diagnosis of sepsis in burn patients: a retrospective study. Burns 2017;43:1427-34

38. Neely AN, Fowler LA, Kagan RJ, Warden GD. Procalcitonin in pediatric burn patients: an early indicator of sepsis? J Burn Care Rehabil 2004;25:76-80.

39. Bargues L, Chancerelle Y, Catineau J, Jault P, Carsin H. Evaluation of serum procalcitonin concentration in the ICU following severe burn. Burns 2007;33:860-4

40. Seoane L, Pértega S, Galeiras R, Astola I, Bouza T. Procalcitonin in the burn unit and the diagnosis of infection. Burns 2014;40:223-9.

41. Lavrentieva A. Diagnostic value of procalcitonin in burn septic patients. Burns 2014;40:1239-40.

42. Paratz JD, Lipman J, Boots RJ, Muller MJ, Paterson DL. A new marker of sepsis post burn injury?* . Crit Care Med 2014;42:2029-36.
43. Mann EA, Wood GL, Wade CE. Use of procalcitonin for the detection of sepsis in the critically ill burn patient: a systematic review of the literature. Burns 2011;41:549-58.

44. Ren H, Li Y, Han C, Hu H. Serum procalcitonin as a diagnostic biomarker for sepsis in burned patients: a meta-analysis. Burns 2015;41:502-9.

45. Cabral L, Afreixo V, Almeida L, Paiva JA. The use of procalcitonin (PCT) for diagnosis of sepsis in burn patients: a meta-analysis. PLoS One 2016;11:e0168475.

46. Lavrentieva A, Papadopoulou S, Kioumis J, Kaimakamis E, Bitzani M PCT as a diagnostic and prognostic tool in burn patients. Whether time course has a role in monitoring sepsis treatment. Burns 2012;38:356-63.

47. Mokline A, Garsallah L, Rahmani I, et al. Procalcitonin: a diagnostic and prognostic biomarker of sepsis in burned patients. Ann Burns Fire Disasters 2015;28:116-20.

48. Barrow RE, Spies M, Barrow LN, Herndon DN. Influence of demo graphics and inhalation injury on burn mortality in children. Burns 2004;30:72-7.

49. Mitsuma SF, Mansour MK, Dekker JP, et al. Promising new assays and technologies for the diagnosis and management of infectious diseases. Clin Infect Dis 2013;56:996-1002.

50. Vincent JL. Rapid diagnosis of infection in the critically ill, a multicenter study of molecular detection in bloodstream infections, pneumonia, and sterile site infections. Crit Care Med 2015;43:2283-91.

51. Nunez Lopez O, Cambiaso-Daniel J, Branski LK, Norbury WB, Herndon DN. Predicting and managing sepsis in burn patients: current perspectives. Ther Clin Risk Manag 2017;13:1107-17.

52. Mickiewicz B, Tam P, Jenne CN, et al.; Alberta Sepsis Network. Integration of metabolic and inflammatory mediator profiles as a potential prognostic approach for septic shock in the intensive care unit. Crit Care 2015;19:11.

53. Hazeldine J, Hampson P, Lord JM. The diagnostic and prognostic value of systems biology research in major traumatic and thermal injury: a review. Burns Trauma 2016;4:33.

54. Vincent JL, van Nuffelen, Lelubre C. Host response biomarkers in sepsis: the role od procalcitonin. In: Nicasio M, editor. Sepsis: diagnostic methods and protocols. 1st Ed. New York: Humana Press, 2015. p. 213-224

55. Trásy $\mathrm{D}$, Tánczos $\mathrm{K}$, Németh $\mathrm{M}$, et al. Delta procalcitonin is a better indicator of infection than absolute procalcitonin values in critically ill patients: a prospective observational study. J Immunol Res 2016;2016:3530752. 